\title{
SOBRE LA REDACCIÓN Y DIFUSIÓN DE LAS "ORDINACIONS" DE PEDRO IV DE ARAGÓN Y SUS PRIMEROS CÓDICES
}

\author{
Bonifacio Palacios Martín \\ Universidad Complutense de Madrid
}

\begin{abstract}
SUMARIO
I. El texto de las Ordenaciones y su divulgación: 1. Elaboración del texto. 2. Difusión del texto de las Ordenaciones.- II. Los primeros códices de las Ordenaciones de Pedro IV: 1. El manuscrito "viejo", "el nuevo" y "el de papel" y una traducción castellana. 2. El manuscrito de San Miguel de los Reyes es "el viejo" de los documentos reales. 3. El códice Esp. 99 de la Bibliothèque National de París y el de San Miguel de los Reyes. 4. El códice castellano (Fundación Lázaro Galdeano, ms. R-14.425).
\end{abstract}

\section{El TEXTO DE LAS ORDENACIONES Y SU DIVULGaCIÓN}

\section{Elaboración del texto}

En 1344 Pedro IV el Ceremonioso había ordenado a su escribano de cancillería Mateo Adriá que le tradujese al catalán las Leges Palatinae del reino de Mallorca, que estaban escritas en latín y habían sido promulgadas por su rey Jaime III en 1337, poco antes de que el reino balear fuera definitivamente anexionado a la Corona de Aragón.

\footnotetext{
"Anuario de Estudios Medievales", 25 (1995)
} 
De esa traducción se conserva actualmente una copia manuscrita, en un códice misceláneo de la Biblioteca Nacional de Madrid', que vierte literalmente al catalán el texto mallorquín, con mínimas adaptaciones a la casa real aragonesa.

Contra lo que a primera vista pudiera parecer, esa escasa adaptación no creaba grandes problemas de aplicación a Pedro IV, ya que el origen común de ambas monarquías hacía que coincidieran bastante en sus instituciones y organismos administrativos.

De cualquier forma la traducción de las leyes mallorquinas no satisfizo plenamente a Pedro IV, que sentía de forma especial la necesidad de tener bien ordenado su reino, y especialmente su casa y su persona, desde las cosas más insignificantes a las de mayor importancia, desde las más triviales a las más trascendentes, como podían ser los actos religiosos de su capilla de palacio.

Podríamos indagar por los motivos de esta preocupación casi obsesiva del Ceremonioso, y hallaríamos que obedecía a motivos tanto de tipo teórico como de orden práctico. Entre los primeros había uno que conviene valorar debidamente: la necesidad de tener bien concordada la teoría y práctica de su reino con los principios doctrinales del orden establecido en la Cristiandad, y la necesidad de expresar de forma clara esa concordancia, a fin de hacerla patente a toda la comunidad cristiana, y justificar así en ese foro supranacional la legitimidad de sus reinos, sus conquistas y anexiones y sus actuaciones de gobierno. Todo sistema político busca en principio esta legitimación, por razones de eficacia. Pero en el caso de Pedro IV había, además, otras razones más concretas. La política de expansión mediterránea de la Corona de Aragón, que tanto él como sus predecesores habían practicado, les habían llevado a anexiones territoriales, como la de Sicilia y últimamente la de Mallorca, cuya legitimidad no era muy clara, lo que les hacía especialmente vulnerables, sobre todo ante la instancia pontificia, de la que, por otra parte, Aragón dependía para practicar el acto de legitimación sacrotradicional de la unción y — según el papadode la coronación. En otros casos, como el de Cerdeña, la anexión de la isla se hizo desde el principio bajo fórmulas feudales que reconocían la soberanía pontificia por la que Aragón pagaba un censo a la Santa Sede. Por todo ello

\footnotetext{
'Biblioteca Nacional de Madrid, ms. 956, ff. 12-86. Ver O. SCHENA, Le leggi palatine di Pietro IV d'Aragona, Cagliari, 1983, pp. 30-31 y 40-41.
} 
la monarquía aragonesa debía cuidar al máximo las apariencias y expresar de manera clara su inserción en el discurso político de la cristiandad no sólo de forma jurídico-racional sino también de forma simbólica, desarrollando un ceremonial en el que tanto la persona del rey como cuantos le rodeaban expresaran mediante actos, palabras y gestos esa inserción en el orden cristiano.

Pedro IV parece preocupado por justificar todo, hasta cosas t?.1 inocuas como el uso de correos: "No hay quien dude -afirma en un momento dado- de que el uso de correos es necesario». El rey no olvida que él es la imagen de Cristo Salvador en la tierra, "cuius typum geris in nomine» se dice en las ordenaciones, y que todo el orden de su reino ha de reflejar el orden divino: "Por lo cual y por cuanto en todo y por todo debemos conformarnos con las obras de Aquel, habemos al presente $a$ imitación suya ordenado los oficios de nuestra casa y corte» ${ }^{2}$. Tampoco olvida que al papa corresponde la representación sacerdotal de Cristo en la tierra, por lo que traza una fina raya entre lo espiritual y lo temporal, reservándose lo segundo. Así cree que es competencia del papa la unción de los reyes, pero se atribuye a sí mismo la de la coronación. Bien claro lo expresará en los ritos correspondientes. No menos preocupado se muestra al justificar las ordenaciones de palacio: «Entre los cuidados que un rey ha de tener para la utilidad de la república y la conservación de la autoridad real los que en mayor aprieto han de poner el corazón del príncipe son tener su casa ordenada prudentemente en todo ${ }^{3}$.

Aunque haya en todas estas expresiones cierta parte de retórica, no parece dudoso que también traslucen los temores de la dinastía condal por la precariedad de algunos de sus derechos políticos, lo que les lleva a adaptar y a extremar el orden de su reino y de su persona mediante esta codificación de prácticas rituales y administrativas. Pero no eran ésos los únicos motivos que impulsaron a Pedro IV a escribir sus ordenaciones. Había también una razón de utilidad, por otra parte evidente, sobre todo si tenemos en cuenta los progresos de la burocracia en esa época y el desglose de algunas funciones administrativas. El rey busca orden y claridad en el desempeño de

\footnotetext{
${ }^{2}$ El "manuscrito de San Miguel de los Reyes" de las "Ordinacions" de Pedro IV, II. Estudio, de Bonifacio Palacios Martín, y traducción de Miguel Clemente (año 1562), Valencia, 1994, p. 101.

${ }^{3}$ El "manuscrito de San Miguel de los Reyes", II, p. 159.
} 
éstas, en pro de la eficacia: «Porque entonces serán los oficios bien regidos cuando la orden de diferencia que entre ellos hay fuera guardada...."

Todos estos motivos indujeron a Pedro IV a no contentarse con una simple traducción de las leyes mallorquinas sino a proceder a la reestructuración de las mismas con la ayuda de sus colaboradores. Esa reestructuración, en la que se reelaboraron motivaciones y presupuestos doctrinales, se pormenorizaron oficios y las normas que los regían, dieron lugar al núcleo fundamental de lo que nosotros conocemos como las "Ordinacions" de Pedro IV, pues siempre estuvieron abiertas a la incorporación de nuevas disposiciones del propio rey y de sus sucesores. Fueron promulgadas el 18 de octubre de 1344.

No pasaron muchos años hasta que en 1353 el Ceremonioso procedió también a perfeccionar el ritual de coronación del rey y la reina, ordenando la redacción de dos nuevos capítulos, que fueron desde entonces incorporados a las Ordenaciones. Se trata de un ceremonial mucho más ampuloso y completo que los existentes hasta entonces. Pero tampoco en esta ocasión el rey partió de la nada. Por el contrario, en la nueva redacción permanecen claramente identificables las distintas fuentes en que hasta entonces se había inspirado el ritual aragonés. Estas fuentes fueron: un ordo borgoñón, conservado con abundantes e interesantísimas notas marginales en un pontifical de la catedral de Huesca ${ }^{5}$; uno o varios ordines o ceremoniales imperiales, uno de los cuales pudo ser el ms. 39-12 de la catedral de Toledo, llevado probablemente a Zaragoza para la coronación de Alfonso IV el Benigno por su hermano Juan, arzobispo toledano; y probablemente las notas de la ceremonia que se organizó en 1328 para la coronación de Alfonso IV el Benigno, que estuvo dirigida por el infante don Pedro, conde de Ribagorza, tío de Pedro IV, y personaje muy influyente en el ceremonialismo del rey ${ }^{6}$. En realidad para Pedro IV se había hecho ya una primera redacción en lengua aragonesa, destinada a su coronación, que tuvo lugar en 1336. No lleva la parte relativa a la coronación de la reina, pues entonces

${ }^{4}$ El "manuscrito de San Miguel", II, p. 101.

${ }^{5}$ Archivo-Biblioteca del cabildo de Huesca, códice 10, ff. 60-68.

${ }^{6}$ B. Palacios, La coronación de los reyes de Aragón (1204-1410). Aportación al estudio de las estructuras políticas medievales, Valencia, 1975, pp. 126-130, 209-222, 233-236 y 259269. 
Pedro no estaba casado y, por tanto, no era necesaria ${ }^{7}$. Pero no satisfecho con ella, y ante la necesidad de completarla con la parte de la reina, el 20 de enero de 1353, estando en Valencia, ordenó a su cancillería que se procediera a esta nueva redacción más solemne, que por cierto iba a ser la última que se hiciera para la monarquía aragonesa. El real decreto figura al comienzo del ceremonial. El trabajo debió hacerse con rapidez pues el 8 de agosto, también desde Valencia, se envió una versión latina del mismo al arzobispo de Zaragoza - que es quien debía oficiar en las coronaciones, como ahí se dice- para que la copiasen en un buen códice de la catedral cesaraugustana, de forma que se asegurara su conservación ${ }^{8}$.

En los años siguientes a estos hechos, la atención del Ceremonioso no se apartó de sus ordenaciones. El rey siguió revisando su funcionamiento en la práctica, y el resultado fue la introducción de modificaciones en algunos cargos con la consiguiente repercusión en el texto. Esta labor se fue reflejando especialmente en un manuscrito de papel que Pedro IV tenía para su uso particular, manuscrito dado por desaparecido desde el siglo XVIII, y que no hace mucho ha sido localizado por $O$. Schena y J. Trenchs, que dan cuenta de él en un trabajo reciente ${ }^{9}$. Ese texto aparece con abundantes correcciones y apostillas, algunas de la propia mano del rey.

\section{Difusión del texto de las Ordenaciones}

Desde el primer momento las ordenanzas palatinas y el ceremonial de coronación de los reyes comenzaron a correr juntos en las múltiples copias que se hicieron (con la única excepción del texto latino del ceremonial de coronación), como si de una única obra se tratara. Y no otra debió ser la intención de su inspirador, Pedro IV el Ceremonioso.

La proliferación de copias obedeció, entre otras razones, a exigencias de uso, ya que se trataba de normas de tipo práctico y aplicación constante. En ellas se establece que cada oficial debía tener una copia del epígrafe

\footnotetext{
${ }^{7}$ Se conserva en manuscrito único en la Biblioteca del Escorial, Et, III, 3. Lo publicaron M. Salvia y P. SÁINZ DE Baranda, "Colección de Documentos Inéditos para la Historia de España", XIV, Madrid, 1894, pp. 556-568.

${ }^{8}$ Se conserva en el Archivo de la Seo de Zaragoza, Cantoral grande, ff. 324-331.

${ }^{9} \mathrm{O}$. SCHENA y J. TREnCHS, Le Leggi (sic) palatinae di Giacomo III de Maiorca nella corte di Pietro IV d'Aragona, "XIII Congreso de Historia de la Corona de Aragón, Comunicaciones, III", Palma de Mallorca, 1990, pp. 111-119. El manuscrito se conserva en la Biblioteca de la Facultad de Derecho de la Universidad de Valencia, sin número.
} 
relativo a su oficio ${ }^{10}$. Pero el usuario principal fue el rey y su corte. Esta era parcialmente itinerante. En 1277 Pedro III había establecido su rotación obligatoria a lo largo del año por los tres territorios que constituían el núcleo fundamental de la Corona: Aragón, Cataluña y Valencia. El rey debía residir cuatro meses al año en cada una de ellas ${ }^{11}$. Y aunque en la práctica ese ritmo de itinerancia no se cumpliera al pie de la letra, en la organización de la casa real se operó un reajuste de instituciones y de todo lo relativo a la instalación del rey en los respectivos palacios de las capitales de esos tres territorios: Zaragoza, Barcelona y Valencia ${ }^{12}$. Al promulgar Pedro IV sus Ordenaciones parece lógico pensar que se preocupara de enviar una copia decorosa a cada uno de esos palacios, para que fueran en ellos el referente figurativo y gráfico del orden que el rey deseaba imponer en su casa y en sus reinos. Es una preocupación que el rey transmitió a sus descendientes. Martín I el Humano censurará más tarde a su hijo Martín el Joven, rey de Sicilia, por no tener organizada su casa «ab aquella ordenada e reglada manera que deu e pertany de Rey» ${ }^{13}$. El propio Pedro IV llevó, al parecer, una copia de las ordenaciones a Cagliari cuando, en la expedición de 13531354, se ocupó de reorganizar la administración real en la isla. Otro tanto hizo Alfonso V el Magnánimo en 1417, en la expedición que preparó a Sicilia, en la que se hizo acompañar, entre otros libros, por un ejemplar de las ordenaciones, de las que posteriormente se hicieron otras copias en la corte de Nápoles ${ }^{14}$. ¿Envió también Pedro IV un ejemplar de sus ordenaciones a sus tres palacios residenciales? El hecho de que en la actualidad se conserven tres ejemplares ricamente miniados, con las armas reales, e históricamente ligadas a las ciudades de Barcelona, Valencia y Zaragoza, nos ha llevado en otro trabajo a pensar que así habría sido, y que esos tres ejemplares, que guardan un gran parecido entre sí, se habrían copiado con

${ }^{10}$ El "manuscrito de San Miguel de los Reyes", II, pp. 104, 124, 143, etc.

"Ver "Colección de Documentos Inéditos del Archivo de la Corona de Aragón", VI, publicado por D. Próspero de Bofarull y Mascaró, Barcelona, 1850, pp. 15-16.

${ }^{12}$ El "Manuscrito de San Miguel de los Reyes", II, pp. 101.

${ }^{13}$ Archivo de la Corona de Aragón, Cancillería, reg. 2299, f. 83.

${ }^{14}$ O. SChEnA, Le leggi, p. 63. R. D’Alos, Documenti per la storia della Biblioteca d'Alfonso il Magnanimo, "Miscellanea Francesco Ehrle - Studi di Storia e Paleografia", V, Roma, 1924, pp. 390-406. Ver también T. DE MARINIS, La biblioteca napoletana dei re d'Aragona, II, Milán, 1917, 118 ss. 
esa finalidad ${ }^{15}$. Hoy, en cambio, considero más que probable que al menos uno de ellos, el que iría muy pronto a parar a Zaragoza, se copió con una intención bien distinta, como veremos más adelante. Aunque a la postre los tres se destinaron al servicio de la casa real en esas tres ciudades.

Otra razón que multiplica los ejemplares de las ordenaciones y complica su contenido es el carácter abierto de las mismas. Como ya hemos adelantado, en los años que siguieron a su promulgación, los reyes modificaron algunos oficios $\mathrm{y}$, por consiguiente, la normativa que los regulaba. Así en 1355 se creó el oficio de protonotario y en 1361, el de lugarteniente del mismo. Pero, sobre todo, se siguieron dando normas nuevas que se añadían al núcleo promulgado por Pedro IV y se copiaban unas veces en los códices ya existentes, aprovechando los espacios vacíos, o bien añadiendo cuadernillos o haciendo copias nuevas ${ }^{16}$.

El reino de Aragón también mostró su interés por poseer un ejemplar completo de las ordenaciones. Hemos mencionado ya la copia latina de la parte relativa a la coronación, existente en la Seo de Zaragoza.

Andrés de Uztarroz en una nota a la edición del libro de Jerónimo Blancas sobre las coronaciones ${ }^{17}$ afirma que la ciudad tenía copiado en su Libro de Privilegios una traducción al aragonés hecha por Antinch de Bages y, por tanto, del siglo XV. No hemos podido localizarla. En el siglo XVI tanto Zurita como Blancas debieron poseer copias completas de las ordenaciones. El segundo transcribió en su libro de las coronaciones una traducción castellana de la parte relativa a la coronación. En algún momento pensamos, inducidos por la nota de Uztarroz, que podría ser la misma que poseía el concejo zaragozano. Hoy podemos demostrar que es copia de una traducción que por orden de Pedro IV se hizo para el rey de Castilla, de la que enseguida trataremos. En la Biblioteca Nacional de Madrid se conserva un códice en catalán del siglo $\mathrm{XVII}^{18}$, copiado por Miguel de Segovia,

${ }^{15}$ El "Manuscrito de San Miguel de los Reyes", II, Estudio, p. 48.

${ }^{16}$ Son muchos los manuscritos que contienen normas de reyes posteriores. El libro de $\mathrm{O}$. Schena ya citado permite comprobarlo. Un ejemplo puede ser el ms. Esp.- 62 de la Biblioteca Nacional de París que, además de las ordenaciones del Ceremonioso (ff. 1-138 v.), lleva ediciones de la reina doña Leonor, Pedro IV, Martín I, Juan I, Alfonso V, e incluso una del primogénito, duque de Gerona, de 1382 (ff. 140-198).

${ }^{17} \mathrm{~J}$. BlanCAS, Coronaciones de los serenísimos reyes de Aragón... Publícalo el Doctor Juan Francisco Andrés de Uztarroz con algunas notas, Zaragoza, por Diego Dormer, 1641, p. 117, nota D.

${ }^{18}$ Biblioteca Nacional de Madrid, ms. 996. 
habitante de Zaragoza y notario público del reino, que usó como modelo otra copia anterior, obra de Jerónimo Lizana.

Las relaciones de la Corona de Aragón con la de Castilla también dieron ocasión a que se produjeran copias de las ordenaciones. En 1383 el rey de Castilla Juan I pidió al de Aragón un ejemplar de las mismas ${ }^{19}$. Como tendremos ocasión de comprobar, el ruego fue atendido. A raíz de la unión de Castilla y Aragón también se realizaron nuevas copias. Bajo Felipe II, en 1562, en el contexto de la confrontación con el foralismo aragonés, se hizo una en catalán y otra en versión castellana (obra del protonotario aragonés Miguel Clemente), dedicada a la educación del infortunado príncipe don Carlos ${ }^{20}$.

En época más moderna las tendencias regalistas de algunos monarcas y de sus consejeros dieron pie al traslado a Madrid de aquellos documentos que afectaban de cerca a la realeza. Así hacia 1782 el conde de Floridablanca solicitó un manuscrito custodiado en el Archivo de la Corona de Aragón, que llevaba anotaciones de la propia mano del rey, del que, antes de abandonar el archivo, se hicieron dos copias: una quedó en él ${ }^{21}$, y la otra se envió a Floridablanca, según testimonio de Próspero de Bofarull, quien en 1850 editó la copia que quedó en Barcelona, y afirma que el manuscrito apostillado de mano del rey fue enviado también a Floridablanca en $1787^{22}$. Durante mucho tiempo este singular ejemplar ha estado desaparecido, hasta su reciente localización por Schena y Trenchs, como ya se ha dicho.

\section{LOS PRIMEROS CÓDICES DE LAS ORDENACIONES DE PEDRO IV}

Es lógico pensar que las primeras copias de las ordenaciones salieron de la misma cancillería real de Pedro IV. La documentación cultural conservada en el Archivo de la Corona de Aragón, que ha sido ampliamente

\footnotetext{
${ }^{19}$ Archivo de la Corona de Aragón, Cancillería, reg. 1287, f. 2.

${ }^{20} \mathrm{La}$ versión catalana estaba en la Biblioteca del Escorial, h.II.9, donde sigue la traducción de Miguel Clemente, h.II.10, recientemente publicada en El manuscrito de San Miguel de los Reyes, II, pp. 95-232.

${ }^{21}$ Archivo de la Corona de Aragón, Casa Real, reg. 1529b.

${ }^{22}$ Colección de Documentos inéditos de la Corona de Aragón, V, Barcelona, 1850, p. 6.
} 
divulgada tras su publicación por Rubió y Lluch y Amada L. de Meneses ${ }^{23}$ así lo confirma. Son muchos los documentos ahí recogidos que nos hablan de la actividad copista y, a veces, traductora llevada a cabo por la cancillería real en la época de Pedro IV. Aunque no estará de más observar que la cancillería es con frecuencia sólo la promotora de un trabajo en el que cooperan escribanos e iluminadores de distintas procedencias. Centrándonos en el tema de las ordenaciones queremos recordar solamente algunos documentos que se refieren a ellas y a su copia o reproducción en la cancillería. Será el punto de partida para tratar de identificar algunos de los códices actualmente conservados.

\section{El manuscrito "viejo", "el nuevo" y "el de papel".., y una traducción castellana}

Dejando de lado otros documentos que en su momento consideraremos, fijémonos en un par de ellos que consideramos clave. El 3 de marzo de 1357, estando el rey en Zaragoza, se dirige a Jimeno de Monreal, escribano de su cancillería, en estos términos: «Molt nos maravellam com no havets expatxat lo libre nou de la nostra ordinacio de casa nostra, ne adobat lo veyll, ne tramés lo de paper, lo qual tenits per exemplarm. Seguidamente le ordena que los acabe cuanto antes y que los trasmita a la reina doña Leonor, y le comunica que ha dado orden de que si hay que pagar algo más que se pague. Un mes más tarde, estando en Zaragoza, ordena que los dos ejemplares en pergamino, el nuevo y el viejo, sean enviados al archivo de Barcelona, y el de papel le sea remitido directamente a é ${ }^{24}$. Años después, el 5 de febrero de 1384, estando en Monzón, encarga diez docenas de buenos pergaminos para un traslado de las ordenaciones que le ha pedido su yerno, el rey de Castilla Juan I. Por otro documento del 27 de junio de 1386 sabemos que dichas ordenaciones habían sido ya enviadas al castellano ${ }^{25}$. En resumen, por estos datos sabemos que de las ordenacio-

${ }^{23}$ A. RUBIó Y LLUCH, Documents per l'historia de la cultura catalana mig-eval, 2 vols., Barcelona, 1908 y 1921; A. LÓPEZ DE MENESES, Documentos culturales de Pedro el Ceremonioso, "Estudios de Edad Media de la Corona de Aragón", V (Zaragoza, 1952), pp. 669-771.

${ }^{24}$ A. Rubió y Lluch, Documents, I, núm. 179, p. 182, y núm. 181, p. 183.

${ }^{25}$ A. RuBió Y LluCh, Documents, I, núm. 350, p. 318; II, núm. 304, p. 295. 
nes de Pedro IV se hicieron antes de 1358 al menos tres copias en pergamino y una en papel. la cual solía llevar el rey consigo en sus desplazamientos.

La cuestión es si se pueden identificar estas cuatro copias salidas de la cancillería real con alguno de los múltiples manuscritos que actualmente se conservan. El interés de esta averiguación no es pequeño. En primer lugar porque arrojaría mucha luz para datar y explicar muchos pormenores de la composición de los códices y permitirá en algunos casos identificar a las personas que intervienen en la obra como copistas, iluminadores y traductores. En segundo lugar porque estos datos proporcionarían un punto de partida firme para cuando se acometa la tarea de hacer el stemma de las múltiples copias que se conservan.

En relación con el conocimiento de esta tradición manuscrita hay que hacer constar, antes de pasar adelante, los avances que se han producido en los últimos tiempos, en los que han sido de nuevo localizados algunos códices que se daban por desaparecidos. El elenco más completo de los mismos lo proporciona $O$. Schena ${ }^{26}$, quien realiza un primer intento de clasificación de los mismos. A los allí señalados hay que añadir otros hallazgos posteriores. "Hallazgo" relativo es el de una versión castellana de la parte de la coronación del rey y la reina, del que la mencionada autora se hace eco, y que tras desaparecer durante la guerra civil, ha regresado ya a su lugar de pertenencia, la Colección Lázaro Galdeano, de Madrid ${ }^{27}$. Hallazgo más importante, el de un manuscrito en papel del siglo XIV, en la Biblioteca de Derecho de la Universidad de Valencia, llevado a cabo por la propia O. Schena y J. Trenchs, quienes lo identifican con "el de papel" de Pedro $\mathrm{IV}^{28}$.

A pesar de estos avances en la localización de copias de las ordenaciones la opinión de los historiadores más especializados se muestra bastante reticente ante la posibilidad de identificarlas con las que se citan en los documentos de Pedro IV. Tanto P. Bohigas como O. Schena (cuya argumentación veremos más adelante) consideran que hay serias dificultades

\footnotetext{
${ }^{26}$ O. SCHENA, Le Leggi palatine, pp.38-67.

${ }^{27}$ Está actualmente en la Biblioteca de la Fundación Lázaro Galdeano, Madrid, ms. R14.425. Ha sido publicado: Ceremonial de la Consagración y Coronación de los Reyes de Aragón, Zaragoza, Diputación general de Aragón, 1991. Vol. I, facsímil; vol. II, estudios de A. San Vicente Pino, M. Agudo Romero, J.M. Enguita y V. Lagüens García, A. Sesma Muñoz y B. Palacios Martín.

${ }^{28}$ O. SChena y J. Trenchs, Le Leggi palatine di Giacomo III de Maiorca, p. 116.
} 
para esa identificación, dada la discordancia que a su entender existe entre los datos que proporcionan los documentos y el examen de los propios códices.

Sin embargo, aún respetando la acreditada opinión de estos autores, pensamos que esa identificación sí es posible. La hipótesis que vamos a tratar de comprobar en las páginas siguientes es ésta: 1. El manuscrito "de papel" que Pedro IV llevaba consigo y que sirvió de modelo para corregir "el viejo" y copiar "el nuevo" es el que recientemente ha sido localizado por O. Schena y J. Trenchs en la biblioteca de Derecho de la Universidad de Valencia. 2. El "viejo" que corregía en 1357 Jimeno de Monreal es el actual ms. 2.633 de la biblioteca de la Fundación Bartolomé March, de Palma de Mallorca, al que en adelante denominaremos el "manuscrito de San Miguel de los Reyes", por haber permanecido durante varios siglos en la biblioteca de este real monasterio valenciano. 3. Identificamos con bastantes probabilidades "el nuevo" de los documentos con el Esp. 99 de la Bibliothèque Nationale de París. 4. Y finalmente se identifica la versión castellana enviada a Juan I de Castilla con el manuscrito R-14.425 de la Fundación Lázaro Galdeano de Madrid, actualmente mutilado, pues sólo conserva la parte relativa a las coronaciones.

Sobre el primer punto de nuestra hipótesis (identificación del manuscrito "de papel" con el hallado en Valencia por el malogrado J. Trenchs y O. Schena) no vamos a tratar aquí porque está siendo estudiado por la investigadora italiana y por F. Gimeno Blay, quien amablemente nos ha puesto al corriente de esta investigación en marcha.

\section{El manuscrito de San Miguel de los Reyes es "el viejo" de los documentos reales}

Es un manuscrito en pergamino, con encuadernación moderna, que contiene el texto de las ordenaciones palatinas (ff. I-XCV v.) y de la coronación de los reyes (ff. XCVIII-CXIV v.). Está escrito en letra gótica libraria o redonda, a dos columnas, de una sola mano. Lleva abundantes notas marginales así como añadiduras en la caja de escritura, casi siempre también de una misma mano. Lleva una orla inicial a toda página con figuras humanas en sus medallones, más otras orlas menores y siete capitales también con figuras humanas, y otras cuatro con dibujos geométricos y algún animal, todo ello con fondos de oro. 
La historia del códice es larga y complicada. Nada se sabe de él con certeza hasta 1550, año en el que pasó a la biblioteca del monasterio de San Miguel de los Reyes de Valencia, como parte de la donación que le hizo el duque de Calabria, virrey de Valencia y último heredero del reino de Nápoles de la dinastía aragonesa. ¿Cómo llegó hasta allí? Su presencia en el legado del duque de Calabria movió a pensar a algunos investigadores, como O. Schena, que habría pertenecido a la famosa biblioteca de los reyes aragoneses de Nápoles ${ }^{29}$. Pero cabe también la posibilidad de que el manuscrito estuviera anteriormente en el palacio real valenciano, donde había depositado otros objetos de la casa real, como los libros de la reina doña María, mujer de Alfonso V el Magnánimo, tal como parece deducirse del inventario de los mismos, actualmente conservado en el Archivo del Reino de Valencia ${ }^{30}$. Apoya esta tesis el hecho de que, si bien es cierto que ese manuscrito figura en el inventario de los libros que en 1550 dejó el duque de Calabria al monasterio valenciano ${ }^{31}$, sin embargo no aparece en los inventarios de la biblioteca napolitana ${ }^{32}$ ni se puede identificar con el que en 1417, cuando se preparaba la primera expedición de Alfonso el Magnánimo a Italia, figuraba en un inventario de libros de la Cámara regia, preparados sin duda para su traslado a Sicilia. Pues en la descripción que se hace del manuscrito se dice «en lo principi del libre, en .i. pagina es lo crucifix ab iii. figures de Santa Maria et de sent Johan e antorn del dit crucifix son los .iiii. evangelis, e an l'altre pagina comensa la rubrica en letres bermelles "rubrica del present libre", e en letres negres "del prohemi", e comença lo dit libre en letres d'aur "Ordinacions fetes", e en letres negres "Nos en Pere per la gracia de Deu"...». Y en el manuscrito de San Miguel de los Reyes no existe la primera página que aquí se describe ni «la rubrica del present libre», ni el comienzo del mismo está en letras de oro ${ }^{33}$.

La historia posterior a 1550 nos es más conocida. Según nota del comienzo del manuscrito, escrita en letra del siglo XVIII, fue prestado por

\footnotetext{
${ }^{29}$ Le Leggi palatine, pp. 64-65, nota 76.

${ }^{30}$ Publicado en Inventari dels libres de la senyora donna María, reina de les Sicilies e de Arago, Madrid, imprenta Rivadeneyra, 1872.

${ }^{31}$ Ver Inventario de los libros de don Fernando de Aragón, duque de Calabria, Madrid, imprenta Aribau y Cía., 1875, p. 30, n. 278.

${ }^{32}$ T. DE MARINIS, La Biblioteca napoletana, II, p. 187 y ss., donde se hace referencia a copias de las ordenaciones hechas en Italia.

${ }^{33}$ Ver R. D’Alos, Documenti per la storia, p. 406, núm. 61.
} 
un monje jerónimo, el P. Parga, a Jerónimo Gavea. Parece que fue entonces cuando salió de Valencia, acaso reclamado por las autoridades centrales. Lo único que sabemos con certeza es que a principios del siglo XIX, en los tiempos calamitosos de la Guerra de Independencia, fue llevado a Inglaterra, y que en 1828 lo había adquirido junto con otras bibliotecas españolas el librero Thorpe, quien ese año lo vendió a sir Thomas Phillips. En 1965 salió a subasta en Sotheby de Londres. Lo adquirió la librería H.P. Kraus de Nueva York. En 1973 fue adquirido por Bartolomé March para la Fundacion Juan March, y depositado en la biblioteca Bartolomé March de Palma de Mallorca.

La identificación del códice de San Miguel de los Reyes con "el viejo" de los documentos ofrece algunas dificultades, pero pienso que no son insalvables. En cambio tiene a su favor una serie de argumentos que, si no son del todo demostrativos, proporcionan una prudente seguridad que deja lugar a pocas dudas.

Para empezar está claro que el códice de San Miguel perteneció (igual que "el viejo") a la casa real. Lo demuestra la presencia en su orla inicial de las armas reales aragonesas junto con la cruz de Sobrarbe, distintivo también de esa monarquía. Por consiguiente se puede asegurar que fue copiado, o al menos encargado, por la cancillería real. Por un documento de ésta, de 9 de diciembre de 1352, estando el rey en Valencia, sabemos que en esa fecha ordena que se paguen a Juan Gil de Castiello, escribano real, 600 sueldos barceloneses por la copia de dos libros, «alterum ordinacionis domus nostrae et alterum de astrologia ${ }^{34}$. Si nos atenemos a las leyes de la crítica histórica que aconsejan no multiplicar los entes sin necesidad, debemos suponer en principio que esta copia de las ordenaciones pudo muy bien ser la del códice que en 1357 los propios documentos reales llaman "el viejo". También nos consta por otro documento que el 23 de julio de 1353, otra vez desde Valencia, Pedro IV ordenó que se pagara a Ferrer de Magarola 250 sueldos barceloneses por catorce letras de oro con historias de diversos colores para un libro en pergamino de las ordenaciones ${ }^{35}$. Parece lógico suponer que, no habiendo en los registros de la cancillería más noticias de otras copias en pergamino y con miniaturas anteriores a 1357, el

${ }^{34}$ A. RUBIÓ y Lluch, Documents, I, n. 164, pp. 164-165.

${ }^{35}$ A. RUBIÓ y LlUCh, Documents, II, n. 107, p. 101. 
manuscrito miniado por Ferrer de Magarola sea el mismo que copiaba Gil de Castiello, y que uno y otro sean "el viejo" de 1357.

Pero hay dos dificultades para esta última identificación. La primera es que el manuscrito del que hablan los documentos estaba ya copiado en diciembre de 1352 y la ordenación de las coronaciones no se promulgó hasta enero de 1353. Luego al estar ésta contenida en el manuscrito "viejo" parece obligado pensar que éste no pudo ser el mismo que un mes antes acababa de escribir Juan Gil de Castiello. Pero dada la escasa diferencia de tiempo (poco más de un mes) y dado que la confección del ritual de la coronación debió comenzar algún tiempo antes, se puede suponer en buena lógica que, dentro de un plan preconcebido, se había previsto ya la copia de las ordenaciones de la coronación después de las de palacio. Lo contrario, es decir, un códice en pergamino y miniado, mutilado de la segunda parte de las ordenaciones que se estaba concluyendo, carecería de sentido por completo. Parece mucho más lógico y normal suponer que fuese el mismo escribiente el que en 1353 copiase esa segunda parte y que fuera entonces miniado - todo él- por Ferrer de Magarola.

Pero si, como nosotros creemos, se identifica el códice "viejo" o de San Miguel de los Reyes con el miniado por Ferrer de Magarola, vuelve a surgir otra dificultad, derivada esta vez del número de sus miniaturas. Cree el investigador catalán Pere Bohigas que no puede tratarse de la misma obra ya que el trabajo que se paga a Ferrer de Magarola es por catorce letras de oro con figuras, mientras que el códice de San Miguel de los Reyes (que él llama "Phillips") sólo tiene once ${ }^{36}$. Sin embargo, y a pesar de lo exacto de su observación, creo que es posible obviar esta dificultad mediante una correcta exégesis de las palabras del documento («XIV lletres d'or... ab istòries de diversos colors»). Vaya por delante que ningún códice de los que se conservan -salidos o no de la cancillería - contiene exactamente las catorce letras historiadas de que habla el documento. En cambio todo se arregla si damos a esa cifra no un sentido literal sino un sentido profesional y lo interpretamos en el contexto económico (pago de un trabajo) en el que se produce. En el lenguaje profesional de aquella época se solía distinguir entre el trabajo de historiar, es decir, de ilustrar el texto con escenas históricas, a veces con fondos dorados («pro miniatura litterarum cum figuris

\footnotetext{
${ }^{36} \mathrm{P}$. Bohigas, El manuscrit Phillips de les "Ordinacions del rey Pere", "Cuadernos de Arqueología e Historia de la Ciudad", XII (Barcelona, 1968), pp. 109-111.
} 
et auro», dicen los textos de la época), obra generalmente de pintores, y el trabajo secundario de colorear y adornar las letras y los márgenes del texto con roleos a pluma, obra de los mismos calígrafos que copiaban el manuscrito, que a veces recibió el nombre de iluminar (aunque este término nunca perdió del todo el significado general que incluía ambos tipos de trabajo). Esta terminología se extendió desde el siglo XIV por Francia, Italia y España ${ }^{37}$. En el texto que nos ocupa es evidente que se está empleando el primer sentido, es decir, el de un trabajo con historia y fondos de oro. El problema es que se habla de catorce letras de oro, lo que parece sugerir que se trata de catorce capitales y, efectivamente, ni en el códice de París ni en el de San Miguel de los Reyes son catorce las capitales historiadas con figuras. Pero fijémonos en el códice de San Miguel de los Reyes (único miniado que en nuestra hipótesis existía entonces): solamente lleva siete «letras de oro con historias», en el sentido literal de esa expresión (folios 1, $2,20 \mathrm{v} ., 35 \mathrm{v} ., 52 \mathrm{v} ., 98$ y 110). Pero junto a ellos hay además otras cuatro que, sin llevar figuras humanas, están también iluminadas con oro y con figuras geométricas y - la última - con figura de animal (folios 59v., 62, 67 y 80). Con fondos dorados están también las diversas orlas que adornan múltiples hojas del manuscrito. En la más importante de ellas, la que está en el folio 1, hay, además, inscritos tres medallones en el centro de sus laterales superior, inferior y derecho con figuras humanas similares en tamaño y en factura a las de las letras capitales. A la hora de evaluar económicamente este trabajo es más exacto decir que había catorce letras historiadas que no siete u once. De manera que nada se opone a suponer que el códice de San Miguel de los Reyes pueda ser el que en 1353, ya con la parte relativa a las coronaciones incorporada, ilustró el pintor Ferrer de Magarola.

De lo que no hay ninguna duda es de que el códice de San Miguel de los Reyes es "el viejo" ("lo veyll") de los documentos cancillerescos. Por ellos sabemos que en 1357 el rey, que después de 1353 no había cesado de introducir correcciones y apostillas a las "Ordinacions", había entregado a Jimeno de Monreal un códice "viejo", para que incorporara en él todos esos cambios y añadiduras, al tiempo que le había encargado la copia de otro nuevo. Por consiguiente el viejo habría de ser, caso de conservarse, un

${ }^{37}$ J. Domínguez Bordona, Diccionario de iluminadores españoles, Madrid, 1957, p. 13; A. VILlalva Dávalos, La miniatura valenciana en los siglos XIV y XV, Valencia, 1964. Cit. por M. SMEYERS, La Miniature, en Typologie des sources du Moyen Age occidental, fasc. 8, Brepols Turnhout, 1974, pp. 18-19. 
manuscrito lleno de raspaduras, correcciones y añadiduras, que reflejasen la acción correctora que sobre él había ordenado hacer el monarca. ¿Responde el de San Miguel a estas características? Así es, en efecto.

Se trata de un códice lleno de raspaduras, espacios reescritos, añadiduras en la caja de escritura y en el margen, al que incluso se ha cortado alguna hoja para sustituirlas por otras, siempre coincidiendo con aquellos capítulos de la obra que fueron objeto de mayores modificaciones por parte del rey y sus colaboradores:

Así, entre los folios 37 y 38 (de una enumeración posterior), se encuentra un folio en blanco, que en su cuadernillo dobla con el 35 , al que está pegado. No hay duda de que se cortó en folio original, en el que empezaba el capítulo dedicado al canciller (que falta), para copiarlo de nuevo con las modificaciones exigidas por la introducción de nuevos cargos en la cancillería. Pero no llegó a copiarse, acaso por las prisas que Pedro IV metió al copista Jimeno de Monreal.

Las correcciones y añadiduras van con frecuencia en el margen del folio correspondiente. Pero cuando se trata de innovaciones más extensas lo normal es que se raspe el texto modificado y se reescriba en ese espacio el nuevo. Pueden verse ejemplos de ello en los folios 3, 9, 14v., 28v., 46v., $53 v ., 56 v ., 59,61,82,91$, etc. En el folio 53v. se borra casi una columna entera, dedicada al oficio del maestre racional. En el $72 v$. hay una nota marginal para adaptar el nombre del emperador al que lo era en el momento de hacerla. Parece de distinta factura que las otras, que mantienen un mismo tipo de letra, y que no parece dudoso que se trata de la de Jimeno de Monreal.

Todo está, pues, a favor de que el códice de San Miguel de los Reyes es "el viejo" que en 1357 arreglaba Jimeno de Monreal y, con bastante probabilidad, es también el que en 1352 copiaba Juan Gil de Castiello (quien pudo añadir seguidamente la ordenación de la coronación) y el que en 1353 había miniado Ferrer de Magarola.

3. El códice Esp. 99 de la Bibliothèque Nationale de París y el de San Miguel de los Reyes

¿Es el Esp. 99 de la Biblioteca Nacional de París "el nuevo" que, según los citados documentos, copiaba en 1357 Jimeno de Monreal?

La verdad es que tal identificación resulta poco menos que necesaria si se acepta que el de San Miguel de los Reyes es "el viejo" mencionado en 
esas fuentes, ya que la similitud entre ambos es extraordinaria, como debía ser la existente entre el viejo y el nuevo, pues, tal como describen las cosas los documentos, el segundo, en cuanto al texto y a la iluminación, tendría que ser una copia exacta del primero.

En efecto, la similitud entre ambos manuscritos, sobre todo en su disposición exterior, es absoluta y unánimemente reconocida. En la miniatura ambos repiten los mismos temas en los mismos lugares, variando sólo ios detalles y la inspiración artística con que se dibujan las escenas y algunos temas ornamentales, como ha señalado Pere Bohigas ${ }^{38}$. Conviene observar, no obstante, al respecto que lo que exhibe el mayordomo en el fol. 2 no es un rollo, como cree Bohigas, sino un bastón, que, según las propias ordenaciones, es el distintivo del mayordomo ${ }^{39}$. También conviene recordar, como diferencia más notable, que el manuscrito parisino lleva en su orla inicial un escudo con la cruz de San Jorge, que no figura en el de san Miguel de los Reyes. Por su parte O. Schena, desde una perspectiva codicológica, y de cara a establecer la filiación de otros manuscritos, coloca a los dos que venimos estudiando a la cabeza de un grupo, y los destaca de los que luego se copiaron por su riqueza y finísima factura, afirmando que las afinidades entre uno y otro son notables ${ }^{40}$.

No obstante, tanto Bohigas como Schena encuentran entre ellos algunas diferencias, que se explicarán desde nuestra tesis de que son "el nuevo" y "el viejo", copiados del "de papel" que Jimeno de Monreal tenía como modelo.

Bohigas señala con acierto ${ }^{41}$ que la figura del rey, que aparece en diversas letras capitales, es juvenil e imberbe en el de San Miguel de los Reyes y con larga barba en el parisino, lo que se explicaría si tenemos en cuenta que, en nuestra hipótesis, este último es, al menos, cinco años posterior al otro, y que en ese tiempo pudo variar el modelo de la iconografía real que solían manejar los pintores. Pero hay otro dato que, tal como lo recoge Schena, parece demostrar lo contrario. En el capítulo Dels escrivans de manament de la nostra scrivania, —-señala la investigadora italiana-, al ofrecer un formulario sobre cómo deben rubricarse las cartas, en la fórmula

\footnotetext{
${ }^{38} \mathrm{P}$. Bohigas, El manuscrit, pp. 102-106.

${ }^{39}$ Ordinacions, f. 3.

${ }^{40} \mathrm{O}$. SCHENA, Le leggi, pp.42-43.

${ }^{41} \mathrm{P}$. BOHIGAS, El manuscrit, pp. 109-111.
} 
de la iussio que sigue al signum regis, la notitia testium y el signum tabellionis, el ms. de San Miguel de los Reyes (o ms. Phillips) transcribe la fórmula ejemplificada de Matheus Adriani, mandato domini regis qui eam vidit, mientras que en el Esp.- 99 se lee Egidius Petri mandato domini regis qui eam vidit. Egidius Petri (Gil Pérez de Buysan) fue notario guardasellos de Pedro IV entre 1336 y 1345. De estos datos deduce Schena que ambos códices proceden de diferente "antígrafo" o arquetipo ${ }^{42}$. Desde luego no se entendería que el códice que se supone más nuevo fuera el único que conservara en la iussio la fórmula más antigua, la que debió figurar en la primera redacción de las ordenaciones. Pero es que las cosas no ocurrieron así, sino de una forma algo más complicada. Si se observa un poco el códice de San Miguel de los Reyes, se ve claramente que en este lugar lleva varias líneas raspadas, pero no tanto que no se pueda leer en una de ellas la iussio antigua («Egidius P[etri] mandato domini regis qui eam vidit»), aunque inmediatamente debajo, sin retoque alguno, viene también la iussio de Mateo Adriá (Matheus mandato domini regis qui eam vidit). No es tan fácil leer lo que se escribió del signum tabellionis, donde se reescribió el relativo a Mateo Adriá ( «Senyal den Matheu... Adriá, protonotari et tinent los segells, etc.» $)^{43}$. Pero hay otro dato muy revelador, que puede aclararnos lo que aquí está sucediendo. En el códice de la Facultad de Derecho de Valencia, que todos suponen que es "lo de paper" que manejaba Pedro IV, hay también señales de haber sido retocado en el signum tabellionis (introduciendo también en él el nombre de Mateo Adriá y transformando el término notari en protonotari) y en la iussio, donde al menos se recalcó el nombre del famoso funcionario de la cancillería de Pedro $\mathrm{IV}^{44}$. De todo ello se deduce que la fórmula antigua no está sólo en el Esp.-99 de París sino también en el de San Miguel de los Reyes y en el de la Facultad de Derecho de Valencia, es decir, en los que nosotros entendemos por "el viejo", "el nuevo" y "el de papel" de los documentos cancillerescos. Por consiguiente hay que suponer que el cambio es posterior a la labor de Jimeno de Monreal, concluida en 1357, y que se hizo sin duda para poner más de relieve en el formulario a la persona y dignidad de Mateo Adriá. En la

\footnotetext{
${ }^{42}$ Afirma la existencia *delle divergenze fra le quali la piu evidente è quella relativa al personagio que compare...», Le leggi palatine, pp. 42-43.

${ }^{43} \mathrm{Cfr}$. el Manuscrito de San Miguel de los Reyes, I. Facsímil, fol. $41 \mathrm{v}$.

${ }^{44}$ Biblioteca de la Facultad de Derecho de la Universidad de Valencia, ms. s/n., fol. LX.
} 
cancilleria no dudaron en hacerlo en los dos manuscritos de menor valor, pero seguramente no se atrevieron a hacerlo en el "nuevo" (Esp.-99 de París) para no enturbiar la belleza y perfección del mismo. ¿Por qué se hizo ese cambio después del trabajo de Jimeno de Monreal y no antes, dado que el ascenso de Adriá a protonotario es de 1355? Aquí cabría imaginar o una pequeña intriga de palacio entre miembros de la cancillería, o simplemente un menor interés en Jimeno de Monreal, que sería prontamente corregido por Adriá o sus amigos de la cancillería en cuanto los manuscritos llegaran a ella.

\section{El códice castellano (Fundación Lázaro Galdeano, ms. $R-14.425)$}

Este manuscrito, del que ya hemos señalado que contiene sólo la parte relativa a las coronaciones, está copiado en lujoso pergamino y adornado con dos espléndidas miniaturas relativas a la coronación del rey y la reina, y con sendas orlas en las que figuran también las armas de la casa real aragonesa. A juzgar por la parte conservada su factura es idéntica a las del Esp. 99 de París y del códice de San Miguel de los Reyes: idéntico lujo, idénticas letras historiadas en la capital inicial de la ordenación de cada coronación, y similares aunque no idénticas orlas (en el códice castellano rodean toda la caja de escritura y llevan también los escudos con las armas reales), resto de capitulares y otras mayúsculas dibujadas con tinta azul y roja, y adornada con roleos y filigranas a pluma, tal como ya se ha descrito. Difiere de los anteriores en que está escrito a toda plana, para dar más realce a la escritura.

Estamos, pues, ante otro ejemplar de lujo salido de la cancillería real aragonesa por encargo del rey. Es además el único ejemplar conservado de época medieval que proporciona un texto de las ordenaciones definitivas de Pedro IV en versión castellana. ¿Con qué objeto y en qué circunstancias se compuso? ¿Quién fue su traductor, su copista e iluminador? ¿Podría ser éste el códice que por orden de Pedro IV se copiaba en 1384 para el rey de Castilla, al que en 1386 ya había sido enviado?

Aunque alguna vez hayamos pensado que el códice de la Lázaro Galdeano podría contener una parte de las ordenaciones de Pedro IV, vertida al castellano (o al aragonés) para uso de su palacio real zaragozano de la Aljafería, hoy pensamos que la mayoría de los indicios coinciden en señalar que se trata de la traducción mandada hacer por Pedro IV para el rey de 
Castilla Juan I. Veamos en primer lugar el perfil de la obra encargada por el Ceremonioso y luego veremos si ese perfil encaja con el manuscrito madrileño.

En documento del 18 de marzo de 1384 el rey paga a Juan de Barbastro tres mil sueldos por el trabajo realizado «in traslatando ordinacionem domus nostre pro rege Castelle» ${ }^{45}$. ¿Cuál es exactamente el trabajo que se paga aquí a Juan de Barbastro: la traducción de la obra o su copia? Seguramente Juan de Barbastro era diestro en ambas cosas, y había recibido del rey encargos de uno y otro tipo. Y también tenemos que el término traslatare (o sus formas romance translatar, translat) podía tener el doble significado de traducir o copiar. Está claro, por ejemplo, que cuando el mismo Pedro IV quiso que Mateo Adriá le tradujese las Partidas de Alfonso el Sabio al catalán, le convoca "per tal que aquells fer tresladar en nostre lenguatge ${ }^{46}$. Que en este caso se trata de la traducción y no de la copia parece demostrarse por dos argumentos: el precio que se paga por la obra, y que los documentos mencionan a otro copista. En efecto, tal como ya se ha dicho, el rey paga tres mil sueldos, cantidad elevadísima si se compara con los trescientos que se dieron a Juan Gil de Castiello por otra copia de las ordenaciones. Y en cuanto al copista, sabemos también que el encargado por el rey fue Ramón de Monzón. Así, estando en Monzón con fecha del 5 de febrero de 1384 , la misma en que el rey ordenaba que se buscasen diez docenas de los mejores pergaminos, convocó al maestro Ramón de Monzón, "escrivá de letra radona" para que «incontinent" compareciese ante él, cosa que hizo aquel mismo día, encargándole copiar «lo translat del qual devem trametre a nostre car fill lo rey de Castella" ${ }^{47}$.

La documentación real también da cuenta de que el encargado de gestionar la iluminación del manuscrito fue Juan de Barbastro, que tuvo que adelantar los 269 sólidos y nueve dineros barceloneses, que fue lo que $\operatorname{costó}^{48}$.

La cuestión es - repetimos de nuevo- si este códice miniado enviado a Castilla es el que se conserva en la fundación Lázaro Galdeano.

${ }^{45}$ ACA, Cancillería, reg. 1371, f. 6 v. Publicado por A. RuBIÓ y LluCh, Documents, II, p. 273 , núm. 280.

${ }^{46}$ Lo cita R. d'ABADAl, Les Partidas a Catalunya durant l'Edat Mitjana, en "Dels visigots als catalans", II, Barcelona, 1962, p. 341.

${ }^{47}$ A. RUBió y LlUCh, Documents, I, p. 317, núm. 349; y p. 318, núm. 350.

${ }^{48}$ A. RUBIÓ y LlUCH, Documents, II, p. 295, núm. 304. 
Nos inclinamos por una respuesta afirmativa, habida cuenta de las coincidencias del códice matritense con el perfil que resulta de los datos expuestos sobre el ejemplar enviado a Castilla. El códice matritense, en efecto, es un manuscrito producido por la cancillería real, ya que lleva los escudos de la casa real en las orlas y el mismo tipo de miniaturas que los manuscritos salidos de la cancillería real en las capitales que inician el ceremonial de coronación del rey y de la reina, aunque en el estilo haya matices diferentes. Por otra parte, la lengua de la traducción apunta también hacia la zona de Aragón fronteriza con Cataluña. Es «una lengua literaria y cancilleresca de fondo genuinamente aragonés pero con rasgos catalanes y, en menor medida, castellanos», propia del siglo XIV ${ }^{49}$. Esta descripción encaja perfectamente con la personalidad de Juan de Barbastro. Y por último si —como hemos visto- el manuscrito enviado a Castilla estaba escrito por Ramón de Monzón, «escrivá de letra radona», no otra es la escritura del de la Lázaro Galdeano: «una gótica textual de trazado caligráfico que tiende al redondeo (rodona es llamada en el área cultural levantina) que transcenderá a la tipografía manual de los incunables» ${ }^{50}$.

No parece, pues, temeraria la identificación del códice matritense con el enviado al rey de Castilla. Pero ¿cuál fue el destino de esta copia y cómo llegó hasta nosotros? No hemos hallado ningún códice que confirme la noticia de Juan Francisco Andrés de Uztarroz de que la ciudad de Zaragoza tenía en su Libro de Privilegios una traducción de las "ordinacions", hecha por Antinch de Bages, al lenguaje aragonés. Lo que sí parece claro es que el texto de la ordenación de las coronaciones que reproduce Blancas es el mismo que aparece en el códice matritense, sólo que un poco actualizado en algunos términos, según el gusto de la época ${ }^{51}$. En el siglo XVI, cuando escribe Blancas, es muy posible, pues, que el códice matritense estuviese ya en Zaragoza. Por otra parte sabemos que éste, en su forma actual (es decir, sin las ordenaciones de palacio), es del siglo XV, pues a esa

\footnotetext{
${ }^{49}$ J.M. Enguita y V. LAgÜEnS, Aspectos Filológicos, "Ceremonial de consagración y coronación, II, Estudio", p. 84.

${ }^{50}$ A. SAN VICENTE DEL PINO, El códice y su transcripción, en "Ceremonial de consagración y coronación, II, Estudio", p. 9.

${ }^{51} \mathrm{~J}$. BlanCaS, Coronación de los serenisimos reyes de Aragón... Publícalo el doctor Juan F. Andrés de Uztarroz, con algunas notas. Zaragoza, 1641, p. 117, nota D.
} 
época pertenece, según los especialistas, su encuadernación ${ }^{52}$. Que el manuscrito llegó de una forma u otra a Zaragoza se confirma por el hecho de que en 1881 estaba en posesión de don Marcial Lorbés, vecino de Sádaba, y que ese año salió de España para una exposición en Inglaterra, ingresando en 1936 en la colección Lázaro Galdeano. Pero ¿cómo y cuándo llegó a Zaragoza? Una hipótesis, a mi juicio muy verosímil, es que el códice actual volvió a Zaragoza sólo 26 años después de que fuera enviado al rey de Castilla, es decir en 1412, con ocasión de la coronación de Fernando de Antequera. El rey necesitaba un texto castellano de la ceremonia que tenía que realizar en Zaragoza. Y la casa real castellana tenía uno muy adecuado por el lujo con que estaba escrito y miniado. Sería entonces, pues, cuando se le arrancaron las ordenaciones palatinas, que no venían al caso, y se encuadernó la parte de las coronaciones en su forma actual. No cabe la posibilidad de que el códice fuera preparado para la coronación de algún otro Trastámara posterior, porque ninguno se coronó después de Fernando de Antequera.

Concluimos, por tanto, que el ms. 2.633 de la Biblioteca Bartolomé March de Palma de Mallorca (que nosotros llamamos "el manuscrito de San Miguel de los Reyes" por el lugar en el que permaneció durante varios siglos), es con todo probabilidad "el viejo" del que hablan los documentos de Pedro IV, probablemente escrito por Juan Gil de Castiello y miniado por Ferrer de Magarola, y con toda seguridad corregido y puesto al día en 1357 por Jimeno de Monreal. El nuevo es, a todas luces el Esp. 99 de la Biblioteca Nacional de París. El "nuevo" y el "viejo" fueron copiados y corregidos respectivamente sobre "el de papel", que, según los investigadores que han trabajado y trabajan sobre él, es el recientemente descubierto en la Facultad de Derecho de Valencia. En cuanto el códice castellano de la Fundación Lázaro Galdeano es, con muchas probabilidades, el que entre 1384 y 1386 se hizo para el rey de Castilla, probablemente devuelto a Aragón por Fernando de Antequera, que lo usaría en su coronación.

\footnotetext{
${ }^{52}{ }_{\kappa} . .$. entre cubiertas de tabla guarnecida en piel gofrada a la morisca $(375 \times 263 \mathrm{~mm}$.), encuadernación del siglo XV». A. SAN VICENTE PINO, El códice y su transcripción, p. 9.
} 


\section{RÉSUMÉ}

Pierre IV le Cérémonieux avait promulgué ses "Ordonnances" pour ordonner sa maison, sa cour, sa personne (un ordre qui devait le légitimer devant la société chrétienne médiévale), et pour assurer le bon fonctionnement administratif qui l'entourait. Ces objectifs expliquent la multiplication de copies qui se trouvent dans un premier moment de sa chancellerie, et plus tard dans d'autres instances. Le travail est centré dans l'identification, qui n'est pas encore réalisée, de plusieurs manuscrits conservés actuellement et avec lesquels on décrit la documentation royale des archives de la Couronne d'Aragon. Trouvé récemment à la Faculté de Droit de Valencia, "le manuscrit en papier" que Pierre IVe emportait dans ses déplacements, nous identifions "le vieux" de ces documents avec le manuscrit 2633 de la Bibliothèque de la Fondation Juan March de Palma de Mallorca; le "nouveau" probablement avec le Esp. 99 de la Bibliothèque Nationale de Paris et, finalement, le manuscrit $R \mathbf{1 4 . 4 2 5}$ de la Fondation Lázaro Galdeano de Madrid, avec la version castillane que le roi demanda de faire pour Jean I de Castille.

\section{SUMMARY}

Pedro IV the Ceremonious had issued his "Ordinacions" to put his house, his court and his own person in order (an order that should legitimize him in the eyes of medieval Christian society), and to insure that his limited administrative organization functioned properly. These objectives explain the number of copies that appeared in the early days of his chancellery and, as time passed, in other instances. This paper focuses on the identification, not previously accomplished, of various manuscripts still extant with those that are described in the royal documentation of the Archives of the Crown of Aragon. The "paper manuscript" that Pedro IV carried with when he travelled having recently been found in the Faculty of Law of the University of Valencia, we identify the "old" document with ms. 2633 in the library of the Juan March Foundation in Palma de Mallorca; the "new" document as probably Esp. 99 in the Bibliothèque Nationale of Paris; and finally, ms. R-14.425 of the Lázaro Galdeano Foundation in Madrid with the version in Castilian done at the King's orders for Juan I of Castile. 